\title{
Philosophiques
}

\section{Les refigurations de notre expérience du temps}

\section{Marie-Hélène Desmeules}

Volume 41, numéro 2, automne 2014

Centenaire de la naissance de Paul Ricoeur (1913-2013)

URI : https://id.erudit.org/iderudit/1027219ar

DOI : https://doi.org/10.7202/1027219ar

Aller au sommaire du numéro

Éditeur(s)

Société de philosophie du Québec

ISSN

0316-2923 (imprimé)

1492-1391 (numérique)

Découvrir la revue

Citer cet article

Desmeules, M.-H. (2014). Les refigurations de notre expérience du temps. Philosophiques, 41(2), 275-293. https://doi.org/10.7202/1027219ar

\section{Résumé de l'article}

Contre l'idée d'un temps fixe enveloppant notre expérience, les travaux de Paul Ricoeur nous permettent de penser que non seulement notre expérience du temps n'est pas si fixe qu'elle n'y paraît, mais qu'elle est en outre transformée par des refigurations découlant de nos médiations objectives. Ces médiations objectives ne se limitent pas à la narration, mais couvrent également l'amnistie, la prescription, la promesse, le pardon et les objets de notre monde familier. Chacune à leur façon, les médiations examinées opèrent une synthèse du passé, du présent et du futur, au point de bouleverser le temps chronologique et successif du courant de la conscience. Ultimement, nous prendrons les réflexions de Paul Ricoeur comme ressources pour penser les implications éthiques des transformations de notre expérience du temps. Car chaque expérience du temps n'ouvre et ne ferme pas les mêmes possibilités, comme le rapport entre le pardon et l'homme capable devrait le montrer.
Ce document est protégé par la loi sur le droit d'auteur. L’utilisation des services d’Érudit (y compris la reproduction) est assujettie à sa politique d'utilisation que vous pouvez consulter en ligne.

https://apropos.erudit.org/fr/usagers/politique-dutilisation/ 


\title{
Les refigurations de notre expérience du temps
}

\author{
MARIE-HÉLÈNE DESMEULES \\ Université Laval \\ Université Paris 1 Panthéon-Sorbonne \\ marie-helene.desmeules.1@ulaval.ca
}

\begin{abstract}
RÉSUMÉ. - Contre l'idée d'un temps fixe enveloppant notre expérience, les travaux de Paul Ricœur nous permettent de penser que non seulement notre expérience du temps n'est pas si fixe qu'elle n'y paraît, mais qu'elle est en outre transformée par des refigurations découlant de nos médiations objectives. Ces médiations objectives ne se limitent pas à la narration, mais couvrent également l'amnistie, la prescription, la promesse, le pardon et les objets de notre monde familier. Chacune à leur façon, les médiations examinées opèrent une synthèse du passé, du présent et du futur, au point de bouleverser le temps chronologique et successif du courant de la conscience. Ultimement, nous prendrons les réflexions de Paul Ricœur comme ressources pour penser les implications éthiques des transformations de notre expérience du temps. Car chaque expérience du temps n'ouvre et ne ferme pas les mêmes possibilités, comme le rapport entre le pardon et l'homme capable devrait le montrer.
\end{abstract}

\begin{abstract}
Against the idea of a fixed time in which our experience would take place, Paul Ricœur's works allow us to think that our experience of time is not so fixed as it seems, and furthermore, that it is transformed by some refigurations which follow from an objective mediation. These objective mediation is not restricted to narrative, but also covers amnesty, prescription, promise, forgiveness and familiar things. Each examined mediation performs in its own way a synthesis of past, present and futur, to the point of changing the chronological and successive time of consciousness' stream. We will also consider Ricœur's thoughts as a possibility to think ethical implications of transformations in our time experience. Every experience of time does not open and close the same ethical possibilities, as the connection between forgiveness and capable man should show it.
\end{abstract}

Edmund Husserl disait que la forme de la conscience intime du temps «est quelque chose d'absolument fixe: la forme subjective du maintenant, de l'avant, etc. Je n'y peux rien changer ${ }^{1}{ }^{\prime}$. L'idée d'un temps fixe enveloppant notre expérience, toute conscience étant prise dans une suite incessante d'impressions originaires, conduira Husserl à parler du flux de la conscience. Changer cette forme du temps ne serait pas en notre pouvoir. Cet article se propose cependant de remettre en question ce constat. Le temps ayant la forme fixe du flux est-il le seul temps que nous ayons? Sommes-nous contraints à n'avoir qu'une seule expérience temporelle, celle de la succession des impressions présentes venant du futur et sombrant dans le passé ?

1. Edmund Husserl, Idées directrices pour une phénoménologie et une philosophie phénoménologique pures - Livre second: Recherches phénoménologiques pour la constitution [Hua IV], Paris, Presses Universitaires de France (coll. Épiméthée), I996, p. 3 I4 [227-228]. 
Notre expérience du temps n'est-elle pas plutôt aussi le fruit d'autres formes de temps, configurées par nos pratiques et notre agir?

À l'appui du soupçon d'une temporalité qui serait instituée par certaines de nos pratiques, nous trouvons le renfort de Paul Ricœur. Car, avec Ricœur, le primat du caractère successif du temps est remis en question. La temporalité ne se résume plus à un maintenant fuyant laissant continuellement place à un nouveau maintenant parce que les trois extases que sont le présent, le passé et le futur sont refigurées dans des intrications opérées et travaillées par des médiations objectives. C'était ce à quoi ses réflexions sur le récit devaient le porter, Temps et récit se concluant notamment sur les apports de la narration pour la refiguration de l'expérience temporelle. Une partie de notre propos prendra appui sur ce constat, et c'est pourquoi nous examinerons ce que veut dire Ricœur lorsqu'il affirme qu'à partir des préfigurations temporelles de l'action quotidienne, le détour par des configurations narratives permet de refigurer notre expérience temporelle.

Loin de remettre en question cette conclusion de Temps et récit, annonçant par ailleurs les études 5 et 6 de Soi-même comme un autre, nous aimerions cependant suivre un certain glissement dans les textes de Ricœur. Car si parfois la médiation narrative est un guide transcendantal pour notre expérience du temps, elle peut aussi avoir pour effet de l'empêcher, de la convoquer abusivement et de la manipuler. Autrement dit, la médiation n'a pas une fonction unique que l'on pourrait résumer sous l'image de la «refiguration» de l'expérience temporelle. Les fonctions de la médiation narrative sont au contraire plurielles. D'un côté, nous montrerons que l'objet peut servir chez Ricœur de guide transcendantal, usage qu'il reçoit de la phénoménologie husserlienne et qui se fait jour lorsqu'il a confiance envers les médiations objectives. Nous expliciterons cette fonction selon que les médiations objectives indiquent quelque chose, permettent de découvrir ou de faire voir du non-dit, stabilisent l'expérience ou même orientent et proposent un monde que je pourrais habiter. De l'autre côté, nous rendrons manifeste une fonction négative de l'objet. Ce dernier n'est alors plus un guide, mais bien ce dont on devrait se méfier non seulement parce qu'il trompe et favorise la mécompréhension, mais aussi parce qu'il agit à l'encontre, par exemple, d'une temporalité heureuse.

Nous souhaitons traverser ces fonctions que l'on peut rassembler sous le terme «refiguration» et qui suivent l'axe décroissant d'une perte de confiance envers l'objet. Nous voulons ce faisant montrer tout le pouvoir transformant des médiations objectives à l'égard de ce qui pourrait pourtant nous paraître comme le plus fixe, c'est-à-dire le temps lui-même. Nous chercherons ainsi autre chose qu'une simple conclusion sur la visée véritative de la mémoire, qui formulerait un devoir de crainte, de suspicion ou de critique à l'égard des médiations objectives qui masquent ou trompent notre rapport au temps. Notre but sera de dépasser l'idée, que l'on trouve dans $\mathrm{La}$ mémoire, l'histoire, l'oubli, d'une juste et fidèle mémoire contre les abus 
potentiels de la narration. Autrement dit, peut-être que les médiations objectives ne servent pas qu'à refigurer, au sens d'une réitération, une première expérience du temps qui a déjà eu lieu. Peut-être peuvent-elles également transformer notre expérience du temps.

Ce faisant, c'est également le rôle privilégié de la narration à titre de médiation que nous souhaitons contester, même si Ricœur affirme parfois le primat de cette médiation par rapport au temps ${ }^{2}$. Certaines ressources non narratives et souvent éminemment éthiques - comme le pardon ou la promesse - ont pour fonction de transformer notre expérience temporelle. Pardonner ou promettre, c'est notamment instituer une nouvelle expérience temporelle. Nous chercherons donc à ouvrir l'étendue des médiations qui travaillent notre expérience du temps. Cela ne se fera pas sans aborder l'idée d'un temps qui serait plus heureux pour l'homme capable. Cet homme rendu à sa capacité d'agir doit porter l'exemplarité de son passé pour savoir agir à l'avenir. Cela n'est cependant possible que si le passé et le futur sont intriqués dans le présent de telle sorte qu'ils sont ouverts les uns aux autres. Ce que nous nous demanderons pour conclure, c'est si certaines transformations de notre expérience temporelle ne sont pas meilleures que d'autres parce que plus adéquates à cette temporalité de l'homme capable.

\section{Le double mouvement du guide transcendantal}

Nous rendrons manifeste la possibilité pour les médiations objectives de refigurer notre expérience du temps non seulement en la révélant ou en la réitérant, mais surtout en la transformant, en rappelant deux fonctions attribuées par Ricœur à l'objet comme guide transcendantal. Point n'est besoin de rappeler pourquoi et comment Ricœur prône une greffe herméneutique à la phénoménologie husserlienne. Contre une compréhension de soi qui serait immédiate, Ricœur souligne l'importance des médiations objectives et d'une herméneutique appliquée à ces médiations. L'herméneutique complète le rejet d'une philosophie qui serait seulement réflexive pour impliquer à titre de corrélat nécessaire à la subjectivité celui de la mondanéité. Dans notre perspective, cela signifie que réflexivité et mondanéité doivent toutes deux être mobilisées pour comprendre notre rapport au temps, comme en témoigne déjà le phénomène de la mémoire qui semble pourtant au plus près de la possibilité d'avoir un rapport immédiat et strictement réflexif à soi:

Je parlerai de la polarité entre réflexivité et mondanéité. On ne se souvient pas seulement de soi, voyant, éprouvant, apprenant, mais des situations mondaines dans lesquelles on a vu, éprouvé, appris. Ces situations impliquent le corps propre et le corps des autres, l'espace vécu, enfin l'horizon du monde et des mondes sous lequel quelque chose est arrivé. Entre réflexivité et mondanéité, il s'agit bien d'une polarité, dans la mesure où la réflexivité est un trait

2. Paul Ricœur, Temps et récit. Tome premier: L'intrigue et le récit historique, Paris, Éditions du Seuil (coll. L'Ordre philosophique), I983, p. 85. 
irrécusable de la mémoire dans sa phase déclarative: quelqu'un dit «en son cœur» qu'il a vu, éprouvé, appris auparavant; à cet égard, rien ne doit être dénié de l'appartenance de la mémoire à la sphère d'intériorité — au cycle de l'inwardness, pour reprendre le vocabulaire de Charles Taylor dans Sources of the Self. Rien, sauf la surcharge interprétative de l'idéalisme subjectiviste qui empêche ce moment de réflexivité d'entrer en relation dialectique avec le pôle de mondanéité ${ }^{3}$.

Il convient cependant de rappeler à quel titre Ricœur convoque l'objet comme pôle nécessaire à la phénoménologie, et ce, dès À l'école de la phénoménologie. Lorsqu'on relit ses premiers articles consacrés à la phénoménologie husserlienne, force est de constater qu'il y découvre l'objet en tant que guide transcendantal. Il remarque alors que:

[1]'analyse intentionnelle prend toujours pour "guide transcendantal» un objet, un sens, dans lequel se nouent des visées de conscience; elle n'aborde jamais directement la génialité sauvage de la conscience; son rationalisme implicite la porte vers l'un, l'ordre, le système dans quoi elle se dépasse polairement. Quelque unité est toujours le fil d'Ariane dans le divers de la conscience ${ }^{4}$.

Ce guide transcendantal peut être un objet perçu, «la réalité dans son ensemble ", la réalité comme nature, la chose, l'esprit, l'histoire, qu'importe. L'important cependant pour Ricœur est que ces objets ne soient pas mis hors jeu dans l'idéalisme professé par Husserl.

Deux fonctions sont alors reconnues à l'objet en tant que guide transcendantal. Ricœur souligne d'abord que les objets donnent une direction à la recherche phénoménologique parce qu'ils servent de télos à atteindre à travers les couches de constitution de la conscience intentionnelle. En parcourant les diverses couches de la conscience, il s'agit de constituer les objets comme unités de sens prédonnés à titre de télos à l'investigation. Il faut réussir à " les retrouver au terme des intentions de conscience qui s’y déposent ${ }^{5}$ ". Mais les objets ont par ailleurs pour fonction de sauver la conscience de sa dispersion et sa discordance dans le temps — l'objet est «le fil d'Ariane dans le divers de la conscience ». Faisant face au flux incessant de la conscience se dispersant dans des impressions originaires toujours nouvelles, l'objet retient l'attention de la conscience. Car lorsque nous imaginons un flux de conscience dans lequel il n'y aurait que des impressions de sensation et nul objet, lorsque nous nous imaginons dans une cellule d'isolement sans nulle ouverture sur le monde et où nous serions confinés à suivre des sensations obéissant à une succession aléatoire, nous sommes en droit de douter: pourrait-il y avoir

3. Paul Ricœur, La mémoire, l'histoire, l'oubli, Paris, Éditions du Seuil (coll. Points), 2003 , p. 44.

4. Paul Ricœur, À l'école de la phénoménologie, Paris, Librairie philosophique J. Vrin, I986, p. 88-89.

5. Ibid., p. 90. 
rétention, souvenir ou attente? Non si l'on en croit Ricœur, puisque la conscience se disperserait dans l'expérience fragmentée de l'impression toujours présente, sans nul point d'ancrage pour rattraper le souvenir d'une sensation plutôt que d'une autre, sans nulle raison d'en attendre une plutôt qu'une autre, et sans aucun «quelque chose » pouvant maintenir la conscience dans la durée.

Ricœur résume alors les deux fonctions de l'objet par l'image du double mouvement d'une conscience qui se rattache à l'objet:

Ideen II ne travaille pas à dissiper le prestige des idées de réalité, de nature, ni le prestige des sciences objectives de l'homme; bien au contraire, en les enracinant dans un travail (Leistung) de conscience, la phénoménologie les justifie et les dresse vis-à-vis de la subjectivité transcendantale comme les objets qui lui donnent sens, la sauvent de l'irrationalité menaçante d'un flux de conscience non concordant. L'objet, c'est la discordance possible surmontée. Ideen II suggère l'image d'une vaste respiration qui fait alterner un mouvement d'influx: ou de retour réflexif à l'Ego pur de la phénoménologie et un mouvement d'expiration ou d'objectivation par quoi la conscience se stabilise dans du réel, dans des significations unes et dignes d'être dites, dignes du $\lambda \dot{\varepsilon} \gamma \varepsilon ı v$ et du $\lambda{ }^{\prime} \gamma \sigma \varsigma^{6}$.

En tant que télos et unité de sens prédonné, l'objet retient l'investigation dans son retour réflexif vers les couches intentionnelles de la conscience pour ne pas qu'elle s'y abîme. L'objet est aussi, en tant que discordance possible surmontée, ce dans quoi se stabilise la conscience contre sa dispersion dans le flux des impressions présentes, discordantes et simplement successives.

Cette analyse de la double fonction de l'objet ne se limite pas aux textes réunis dans À l'école de la phénoménologie, puisque trente années plus tard nous la retrouvons dans Temps et récit, alors que Ricœur commente les Leçons pour une conscience intime du temps de Husserl et l'expérience temporelle qui en ressort. L'objet temporel perçu et ayant une durée, comme la mélodie, ou même l'objet senti le plus minimal qui soit, comme le son, y sont d'abord les guides directeurs pour l'investigation du temps. Suivant les raisonnements de Husserl, Ricœur remarque que le retour réflexif vers les couches intentionnelles les plus profondes de la conscience ne peut se faire qu'en se raccrochant à l'objet comme télos présumé et à constituer dans l'investigation phénoménologique. La révélation des couches intentionnelles de la conscience ne peut se faire que grâce à l'objet comme télos à l'investigation. Car ce que montrent et révèlent le son et la mélodie en tant qu'objets ayant une durée, c'est que l'analyse phénoménologique des couches intentionnelles de la conscience doit parvenir à constituer leur durée. C'est parce qu'ils se dévoilent comme objets ayant une durée que l'analyse intentionnelle de la conscience portera sur la possibilité de constituer la durée - ce que fera Husserl grâce au présent élargi. Ricœur affirme

6. Ibid., p. 90-9I. 
qu' « [e]n attendant, c'est le tempo-objet en tant qu'objet réduit qui fournit son télos à l'investigation; c'est lui qui indique ce qu'il faut constituer dans la sphère de pure immanence, à savoir la durée, au sens de la continuation du même à travers la succession des phases autres ${ }^{7}$ ». Et Ricœur poursuit en soulignant que, sans objet ayant une durée, nous serions probablement restés dans des considérations portant sur la succession et le flux constant du présent de la conscience. Autrement dit, l'objet devient également ce dans quoi la conscience se stabilise et trouve une autre expérience du temps que celle du divers successif des impressions présentes. L'objet transforme l'expérience du temps chronologique et successif en ouvrant l'attente, le souvenir et la rétention.

On peut certes se demander jusqu'à quel point Ricœur a repris à son compte ce qu'il dit de l'objet comme guide transcendantal chez Husserl. Cela correspond cependant à ses mises en garde contre une réflexion qui ne reconnaîtrait plus la mondanéité, ici l'objet temporel, dans ce qu'elle peut apporter à la compréhension. De plus, cette distinction nous permettra d'éclairer ce que Ricœur entend par refiguration narrative de l'expérience. Car dans Temps et récit, la narration sert à la fois de support pour le retour réflexif vers la compréhension de soi et de fil d'Ariane à travers le divers de la conscience ou, comme le dira Jean Greisch, de gardien de la cohésion de la vie ${ }^{8}$.

\section{La narration comme refiguration de l'expérience temporelle}

Cette double fonction de l'objet comme guide transcendantal nous aidera à comprendre à quels titres la narration refigure notre expérience du temps. Lorsque Ricœur entreprend de penser la médiation entre le temps et le récit, c'est qu'il se propose "de montrer le rôle médiateur de ce temps de la mise en intrigue entre les aspects temporels préfigurés dans le champ pratique et la refiguration de notre expérience temporelle par ce temps construit ${ }^{9}{ }$. En suivant le chemin des préfigurations, configurations et refigurations narratives, Ricœur nous invite à reconnaître que le temps humain ne se gagnerait qu' "articulé sur un mode narratif ${ }^{10}$ ». Quelles sont alors les contributions de la narration pour notre expérience du temps, contributions résumées par le terme somme toute assez ambigu de "refiguration " ? Qu'opère la narration à l'égard des aspects temporels préfigurés du champ pratique?

Précisons de prime abord que, pour répondre à ces questions, nous prendrons principalement pour exemple le récit de fiction qui est, de l'aveu

7. Paul Ricœur, Temps et récit. Troisième tome: Le temps raconté, Paris, Éditions du Seuil (coll. L'Ordre philosophique), I985, p. 43.

8. Jean Greisch, "La "cohésion de la vie". La trace comme effet-signe et l'historialité", Noesis, vol. I, I997, p. 5 .

9. Ricœur, Temps et récit. Tome premier: L'intrigue et le récit historique, p. 87.

10. Ibid., p. 85 . 
de Ricœur, «plus riche en informations sur le temps ${ }^{11}$ » que le récit historique en raison de sa dimension d'irréalité. La fiction ne travaille pas à relier le temps de la conscience sur le temps cosmique ou objectif, mais cela «a pour contrepartie positive l'indépendance de la fiction dans l'exploration de ressources du temps phénoménologique qui restent inexploitées, inhibées, par le récit historique $^{12}{ }$. Il faut dire également que Ricœur utilise la fiction narrative pour répondre aux limites que la phénoménologie pure du temps de Husserl rencontre, en illustrant de façon renouvelée ses thèmes et ses apories par «l'exploration des traits non linéaires du temps phénoménologique $[\ldots]^{13}$ ». Mais la fiction ne sert pas qu'à répondre aux problèmes d'une phénoménologie portant sur le temps. Ricœur explore ses ressources surtout parce qu'elle participe, en tant que narration, à la refiguration de notre expérience en portant au langage un monde fictif d'après lequel le lecteur mesure son propre monde afin de mieux se comprendre ou pour changer son agir ${ }^{14}$.

Qu'entend alors Ricœur par cette refiguration qui doit résulter de la lecture des configurations narratives, et qui fait quitter le niveau prénarratif de l'expérience? Il y inclut autant le pouvoir de révéler que celui de transformer l'expérience prénarrative: la refiguration est «la puissance de révélation et de transformation exercée par les configurations narratives quand elles sont "appliquées" à l'agir et au pâtir effectifss ${ }^{15}$ ». Révélation et transformation que l'on retrouve d'abord dans la fiction, puisqu'elle est à la fois "révélante et transformante à l'égard de la pratique quotidienne; révélante, en ce sens qu'elle porte au jour des traits dissimulés, mais déjà dessinés au cœur de notre expérience praxique; transformante, en ce sens qu'une vie ainsi examinée est une vie changée, une vie autre ${ }^{16}{ }^{\star}$. Ce double pouvoir qui ne se limite cependant pas à la fiction, puisqu'il s'étend selon Ricœur à l'ensemble des récits, dont les récits sur soi. Ces récits sont tout à la fois révélateurs d'une expérience préfigurée, et c'est en ce sens qu'ils n'imposent pas simplement de l'extérieur l'expérience qu'il s'agit de raconter ${ }^{17}$. Et ils la transforment

11. Paul Ricœur, Temps et récit. Second tome: La configuration dans le récit de fiction, Paris, Éditions du Seuil (coll. L'Ordre philosophique), I984, p. 232.

12. Ricœur, Temps et récit. Troisième tome: Le temps raconté, p. I 85.

13. Ibid., p. I9г.

14. Cf. ibid., p. 358-359; Ricœur, Temps et récit. Second tome: La configuration dans le récit de fiction, p. 234 ; Ricœur, Temps et récit. Tome premier: L'intrigue et le récit historique, p. 86.

15. P. Ricœur, "Le temps raconté", Revue de métaphysique et de morale, vol. 89, no 4, I984, p. 437.

16. Ricœur, Temps et récit. Troisième tome: Le temps raconté, p. 229.

17. En ce sens, les configurations narratives répondent à des demandes de récit contenues au niveau prénarratif, et qui peuvent servir "d'instance critique " à l'artifice contenu dans l'art de raconter: Cf., Ricœur, Temps et récit. Tome premier: L'intrigue et le récit historique, p. I 2 et suiv. Sur l'importance de l'expérience vive dans les récits vécus, et ce, contre la fabulation et la création narrative: Cf., Laszlo Tengelyi, L'histoire d'une vie et sa région sauvage, Grenoble, Jérôme Millon, 2005, p. 9 et suiv. 
également, la narration ne sombrant pas dans le cercle vicieux de la simple itération d'une expérience préfigurée ${ }^{18}$.

Un problème s'impose cependant pour notre propos portant sur le temps. Comment cette refiguration de l'agir par le récit peut-elle refigurer notre expérience $d u$ temps? Ce qui saute aux yeux, c'est que Ricœur parle ici d'une refiguration de l'action, et non plus d'une refiguration de l'expérience du temps. Ainsi décrite, la narration semble beaucoup plus avoir une prétention éthique qu'une prétention temporelle. Car si le lecteur peut voir son agir transformé, il n'est pas évident que cela concerne sa temporalité. L'action n'est-elle pas dans un temps qui lui ne change pas? Ricœur est-il en train de négliger la problématique du temps au profit de celle de l'action?

Pourtant, même si la narration semble d'abord refigurer l'agir et le soi, c'est en regard du temps que Ricœur la mobilise à nouveau dans Soi-même comme un autre, établissant clairement le lien entre la mise en intrigue de l'action et la refiguration de l'expérience du temps. Si la narration y refigure notre expérience du temps, c'est parce qu'elle ne confine pas les actions à la temporalité du flux de la conscience, c'est-à-dire au temps de la succession des impressions originaires et ponctuelles. Non pas que nous serions, au niveau prénarratif, prisonniers d'une temporalité ainsi fragmentée. Nos actions s'y élèvent déjà à des extensions temporelles plus vastes lorsqu'elles sont incorporées à des hiérarchies d'unités praxiques ${ }^{19}$. Ricœur cite à cet égard ce qu'il nomme les pratiques (comme un métier, un jeu ou un art), les plans de vie (comme la vie professionnelle, de famille et de loisir) et les idéaux qui valent pour l'ensemble d'une vie. Dans chacun de ces cas, les unités praxiques modifient la compréhension que nous pouvions avoir d'une action isolée, sans se résumer à n'être que de simples additions d'actions dans une chaîne plus ou moins longue ${ }^{20}$.

L'intervention propre à la narration, c'est de mettre en intrigue les actions de ces hiérarchies et de les rassembler sous la forme du récit. Ce faisant, l'expérience temporelle des actions prénarratives est refigurée de deux façons. La narration reprend d'abord de façon mimétique les complexités de l'action, notamment pour faire voir le changement graduel des hiérarchies pratiques qui n'ont jamais pour effet de clore l'agir dans l'unité figée d'un projet valant une fois pour toutes. Le récit redéploie le mouvement et

18. Sur l'accusation de redondance entre la préfiguration et la refiguration: $C f$., Ricœur, Temps et récit. Tome premier: L'intrigue et le récit historique, p. I I 3 -I I 6.

19. Paul Ricœur, Soi-même comme un autre, Paris, Éditions du Seuil (coll. Points), I 990 , p. I 8 I.

20. Cette unité se configure dans une pratique notamment à partir de relations linéaires et coordonnées d'actions (pour cueillir des pommes, il faut d'abord monter à l'arbre), de relations de subordinations entre des actions complexes et des gestes (pour labourer, il faut tirer et pousser), de règles constitutives qui donnent des significations aux gestes (secouer la main vaut comme une salutation dans la société) et d'interactions entre les pratiques (la décision d'un juge par rapport à son exécution par les agents sociaux). Ibid., p. I8 I et suiv. 
l'élaboration des unités pratiques à travers le temps selon l'influence réciproque des idéaux sur les actions et des actions sur les idéaux ${ }^{21}$. Le «re " de la refiguration qu'opère la narration équivaut alors à une itération qui nous permet de voir et de revoir l'élaboration de l'agir et de ses hiérarchies dans le temps, c'est-à-dire dans leur mobilité.

Mais surtout, la narration contribue aussi à ôter le caractère chronologique de l'action. Car, loin de se borner à la structure formelle du flux de la conscience et à la simple succession du présent, la narration participe à une unification temporelle particulière:

[J]e propose de définir la concordance discordante, caractéristique de toute composition narrative, par la notion de synthèse de l'hétérogène. Par là, je tente de rendre compte des diverses médiations que l'intrigue opère - entre le divers des événements et l'unité temporelle de l'histoire racontée; entre les composantes disparates de l'action, intentions, causes et hasards, et l'enchâinement de l'histoire; enfin, entre la pure succession et l'unité de la forme temporelle -, médiations qui, à la limite, peuvent bouleverser la chronologie au point de l'abolir22.

La refiguration narrative défigurerait les traits trop linéaires, objectifs et successifs du temps, possibilité que l'irréalité de la fiction nous avait déjà fait entrevoir.

Comment alors la narration parvient-elle à transformer les traits trop linéaires de l'action? N'est-elle pas elle-même prise dans la suite des phrases et de l'acte de lecture, réduite à ne pouvoir raconter qu'une action à la fois? Ricœur entrevoit le problème dans Temps et récit, mais soutient que le temps de la narration n'est pas que chronologique et successif:

[L]'acte de mise en intrigue combine dans des proportions variables deux dimensions temporelles, l'une chronologique, l'autre non chronologique. La première constitue la dimension épisodique du récit: elle caractérise l'histoire en tant que faite d'événements. La seconde est la dimension configurante proprement dite, grâce à laquelle l'intrigue transforme les événements en histoire. Cet acte configurant consiste à «prendre-ensemble » les actions de détail ou ce que nous avons appelé les incidents de l'histoire; de ce divers d'événements, il tire l'unité d'une totalité temporelle. [...] L'acte de l'intrigue a une fonction similaire, en tant qu'il extrait une configuration d'une succession ${ }^{23}$.

Ricœur poursuit en affirmant que le temps de la narration est une totalité signifiante qui a un point final et qui inverse la flèche naturelle du temps allant du passé vers le futur - la fin s’y lit dès le commencement et le commencement encore dans la fin. Sa solution est donc de montrer que la narration rassemble les actions en une unité intelligible et différente de la suite

21. Ibid., p. I86-I 87 .

22. Ibid., p. I68-169.

23. Ricœur, Temps et récit. Tome premier: L'intrigue et le récit historique, p. I03-104. 
chronologique ${ }^{24}$. Car l'unité temporelle de la narration équivaut à la transformation du "l'un après l'autre" des moments de la conscience en une unité cohérente de l'ordre du «l'un à cause de l'autre ${ }^{25}$. Le fil du déroulement successif des actions et des événements, qui surmontait leur dispersion temporelle par leur simple position chronologique, fait place au fil d'Ariane qu'est l'intrigue, c'est-à-dire au fil permettant aux actions de se stabiliser dans "des significations unes et dignes d'être dites ". Grâce à la mise en intrigue, nous ne faisons pas que suivre les actions, mais nous comprenons également les unités de sens dans lesquelles elles s'inscrivent et qui, en raison de leur cohérence, rendent acceptable et vraisemblable leur déroulement. Parce qu'au final, ce n'est pas la position de l'action dans le cours du temps qui la rattache à l'ensemble du récit, mais plutôt sa contribution à faire comprendre comment et pourquoi «les choses devaient "tourner" comme elles l'ont fait ${ }^{26}$ ». Ainsi, «[c]omprendre l'histoire, c'est comprendre comment et pourquoi les épisodes successifs ont conduit à cette conclusion, laquelle, loin d'être prévisible, doit être finalement acceptable, comme congruante avec les épisodes rassemblés ${ }^{27}$ ». Le temps de la narration n'est donc pas celui, linéaire, du flux de la conscience, mais plutôt celui de la synthèse entre le présent, le passé et le futur éloignés selon leur contribution à la cohérence du récit. Le temps synthétisé par la narration peut alors être celui où le passé chargé d'attentes est compris dans le présent, où le présent et ses attentes sont compris dans le futur, et où le futur révèle rétrospectivement la signification du présent et du passé. Il rassemble ce faisant le passé, le présent et le futur, non pas selon leur position chronologique, mais selon le contenu de sens de moments temporels qui peuvent être très éloignés. Par exemple, dans Mrs. Dalloway, Virginia Woolf y parvenait selon Ricœur en retardant le déroulement de l'histoire par l'intervention de "protentions issues de quasiprésents appartenant au passé dépassé ${ }^{28}$ ». Par un retour aux attentes du passé, les personnages étaient non seulement tirés vers le passé éloigné, mais également vers le présent déjà contenu dans les attentes du passé.

Ce détour par Temps et récit et Soi-même comme un autre nous aura permis de comprendre que la narration refigure non seulement l'action, mais également notre expérience du temps, et ce, parce qu'elle est itération et transformation. Nous avons ce faisant retrouvé la double fonction de l'objet comme guide transcendantal, qui est cependant un peu modifiée. La narration agit à titre de support pour faire voir non plus les niveaux constitutifs

24. Ibid., p. I04-105.

25. Ricœur rappelle, en citant Aristote, que: "[1]'art de composer consiste à faire paraitre concordante cette discordance: le "l'un à cause (dia) de l'autre" l'emporte alors sur le "l'un après (méta) l'autre» ( 52 a I 8-22). C'est dans la vie que le discordant ruine la concordance, non dans l'art tragique» (ibid., p. 72).

26. Ibid., p. 289.

27. Ibid., p. I04.

28. Ricœur, Temps et récit. Troisième tome: Le temps raconté, p. I92. 
de la conscience intentionnelle, mais l'expérience préfigurée qu'elle réitère et révèle par le fait même. C'est la narration comme support pour le retour réflexif vers la compréhension de soi. Et elle est également l'objet dans lequel la conscience se stabilise en synthétisant la discordance et la dispersion de ses extases temporelles. Elle élève les actions à l'unité d'un temps particulier, non chronologique et suivant un autre ordre que la flèche allant successivement du passé au futur. Ce temps travaillé par la narration, c'est celui où le lien entre le passé, le présent et l'avenir dépend de leur intrication dans des unités de sens et de leur contribution au tout vraisemblable et cohérent qu'est l'histoire.

\section{Les refigurations non narratives de l'expérience du temps}

Ce détour par Temps et récit et Soi-même comme un autre devait nous permettre de montrer en quoi la médiation narrative refigure notre expérience temporelle, non pas seulement en l'illustrant ou la réitérant de façon à la révéler, mais également et surtout en la transformant. Nous pouvons parler d'une temporalisation synthétisée par la médiation narrative. Mais, ainsi guidés par la double fonction de l'objet comme guide transcendantal, n'avons-nous pas négligé d'autres fonctions potentielles de la refiguration narrative de notre expérience temporelle? S'il est vrai que la narration transforme notre temporalité, le fait-elle toujours en la stabilisant dans la cohérence, tel un guide positif? N'y aurait-il pas par ailleurs d'autres figures d'une temporalisation qui ne se réalise pas nécessairement par la narration, mais néanmoins par des médiations objectives? Se peut-il alors que ces médiations objectives se retournent contre le soi, subvertissant une expérience temporelle autrement heureuse?

Ricœur explore dans La mémoire, l'histoire, l'oubli de nouvelles transformations que la narration, loin de n'être que concordance d'une discordance possible surmontée, peut exercer sur notre expérience. Il y a alors un risque d'abus de la narration qui se décline selon que la mémoire et l'oubli sont empêchés, manipulés ou obligés. Risque d'abus qu'il faut déjà attribuer au caractère sélectif du récit. Un récit ne peut être exhaustif, et, s'il raconte certaines choses, c'est parce qu'il en oublie également, et il est donc toujours possible de raconter autrement ${ }^{29}$. Risque d'abus qui provient également de la clôture du texte qui contribue à négliger son extratextualité. Car si la lisibilité du récit fait voir ce qui n'est plus ou ce qui n'est pas, cette visibilité se suffit également à elle-même - c'est tout un monde qui est déployé par le texte - et peut se scinder du réel qu'elle devait raconter ${ }^{30}$. La narration tend

29. Ricœur, La mémoire, l'histoire, l'oubli, p. 579 et suiv.

30. Cf., ibid., p. 306; 319; 32I; 34I-342;360-362. 
alors «à faire cercle avec elle-même ${ }^{31}$ », et la cohérence narrative suffit seule à faire croire sans recours au dehors du texte ${ }^{32}$.

Cette explication des abus de la narration laisse cependant l'impression qu'il y aurait des abus seulement relativement à une visée cognitive et véritative de la mémoire et de l'histoire. Et il est bien vrai que dans $L a$ mémoire, l'histoire, l'oubli, Ricœur s'attarde principalement à la prétention de vérité de l'histoire que n'a pas la fiction, et à la distinction entre l'image et le souvenir, le second ayant seul une position de réalité, bien que ce soit celle de l'antériorité ${ }^{33}$. Contre les abus de la narration, Ricœur propose alors un réalisme critique, où le témoignage et la mémoire sont les ultimes gages de la prétention de vérité qui traverse le récit historique et l'identité narrative $^{34}$. Ce faisant, il convoque l'expérience prénarrative de la mémoire à titre de gardienne des abus de la narration, et la refiguration narrative signifie alors itération d'une expérience prénarrative primordiale.

Il convient d'abord de préciser que les abus de la mémoire et de l'oubli n'affectent pas seulement leur portée référentielle, mais également la temporalité qu'ils offrent à notre expérience. La mémoire et l'oubli empêchés correspondent par exemple, à titre de temporalités, à un maintien problématique du passé dans le présent. C'est le cas de la mélancolie, affection de l'homme mature qui voit son désir se porter, encore dans le présent, vers un objet qui n'est plus réel, mais de l'ordre du passé ${ }^{35}$. Le passé ayant été reste alors dans le présent sans porter la marque du ne plus, et il faut un travail de deuil pour qu'il relève enfin de l'antérieur. L'abus par manipulation porte aussi une transformation négative de l'expérience temporelle, puisque les identités fragiles de l'individu et des collectivités sont alors manipulées par trop de mémoire et trop d'oubli ${ }^{36}$. Le devoir de mémoire, lorsqu'il reste dans les limites de l'idée de justice, parvient à rallier passé et futur en retournant la mémoire en projet ${ }^{37}$. Mais il peut à l'inverse sombrer dans l'abus lorsque la frénésie de la commémoration ne laisse plus de place au futur. Elle enferme alors la conscience dans la demande de justice des victimes, et nous contraint à la remémoration de la faute passée sans ouverture vers des projets futurs. Dans tous ces exemples, il y a une synthèse particulière entre le présent, le passé et le futur: le passé ne s'accorde plus en tant qu'antérieur, mais en tant que présent; il n'est revendiqué que s'il est en concordance avec les intérêts présents et sans égard pour ce qu'il pourrait nous dire de plus ou autrement; et il concentre sur lui toute l'attention sans s'accorder un futur. Ces synthèses,

31. Ibid., p. 306.

32. Ibid., p. $3 \mathrm{I} 3$.

33. Ibid., p. 53-54.

34. Ibid., p. 364 . Cf., ibid., p. 647-648.

35. Ibid., p. 89 et suiv.

36. Ibid., p. 98 et suiv.

37. Ibid., p. I07. 
comme le suggère le terme «abus ", transformeraient négativement notre expérience temporelle.

De plus, dans la perspective où nous souhaitons délaisser la question cognitive, la refiguration de la temporalité du sujet ne peut pas équivaloir à une itération d'une expérience préfigurée. En embrassant l'idée d'une temporalité qui doit quelque chose à l'objet à titre de "fil d'Ariane pour le divers de la conscience ", nous voulons trouver, en nous aidant de Ricœur, $c e$ qui travaille cette temporalité. Or, force est de constater que La mémoire, l'histoire, l'oubli contient des réflexions sur des médiations autres que narratives qui refigurent la temporalité du sujet en la transformant.

Deux exemples mineurs contribuent déjà à éclairer notre pratique du temps, soit ceux de l'amnistie et de la prescription. Décrivant les deux exemples, Ricœur affirme:

À la différence de l'amnistie qui, comme il a été montré à la fin du chapitre sur l'oubli, tend à effacer les traces psychiques ou sociales, comme si rien ne s'était passé, la prescription consiste en une interdiction de considérer les conséquences pénales de l'action commise, à savoir le droit et même l'obligation de poursuivre pénalement. Si la prescription a affaire avec le temps, si elle est « un effet du temps» comme le déclare le Code civil, c'est de l'irréversibilité qu'il s'agit: c'est le refus, après un laps d'années défini arbitrairement, de reparcourir le temps en arrière jusqu'à l'acte et ses traces illégales ou irrégulières. Les traces ne sont pas effacées: c'est le chemin jusqu'à elles qui est interdit, ce que signifie le mot "extinction » appliqué aux créances et au droit de poursuite pénale ${ }^{38}$.

Ricœur fait ici référence à un «effet du temps » que la prescription et l'amnistie viendraient entériner, ou pourrions-nous dire réitérer, ce que réaffirme sa note: "[à] la faveur du temps, quelqu'un a peut-être été spolié à un certain moment et un autre amnistié de sa violence originelle ${ }^{39} »$. Mais selon notre lecture, ce qui importe dans ces deux exemples, ce n'est pas l'« effet du temps » que viendraient réitérer l'amnistie et la prescription, mais plutôt comment prescription et amnistie travaillent notre expérience du temps. Car la prescription fait quelque chose. Elle interdit «le chemin» à rebours

38. Ibid., p. 6 Iо.

39. Ibid., p. 6Io. Nous ne souscrivons pas à la lecture de la prescription comme confirmation d'un simple "effet mécanique du temps ». Car, dans ce laps de temps, des choses se sont passées. Pour la prescription acquisitive, on exige par exemple la preuve que, dans ce laps de temps, quelqu'un s'est occupé de bonne foi des biens qu'il tente d'acquérir. Pour la prescription extinctive, le simple passage mécanique du temps ne suffit pas, parce qu'il peut être suspendu lorsque la victime est dans l'incapacité d'agir, notamment par traumatisme. Par ailleurs, ce qui passe dans la durée de la prescription, ce n'est pas que le temps, mais également la possibilité d'établir un lien direct entre la punition et le fautif qui doit être puni. Dans ce laps de temps, il y a présomption que le fautif $n$ 'est plus le fautif parce qu'il est devenu autre, et peut-être meilleur, que celui qu'il était lors du crime. Sauf par vengeance, pourquoi alors punir celui qui, au présent, n'est plus le fautif d'alors? 
jusqu'à l'origine d'une possession ou d'un crime dont les traces sont pourtant encore présentes. L'expérience du temps est refigurée parce que l'origine temporelle est effacée, même si les traces de cette origine sont conservées à titre de nouvelle possession ou de blessure chez la victime. L'amnistie institue quant à elle l'amnésie du passé, c'est-à-dire l'effacement des traces de ce passé dans le présent et le futur ${ }^{40}$. Dans les deux cas, une synthèse particulière de l'expérience temporelle est imposée.

Ricœur ne se limite pourtant pas aux exemples de l'amnistie et de la prescription, et il explore également la promesse et le pardon. Certes, il s'attarde surtout à montrer comment le pardon et la promesse ne sont pas symétriques, alors qu'au contraire Hannah Arendt soulignait comment le pardon permet de délier des conséquences de ses actes celui qui pourra par la suite se lier dans la promesse. Pour Ricœur, une certaine "symétrie entre ces deux "faiblesses" que les choses humaines doivent à leur condition temporelle, irréversibilité et imprévisibilité ${ }^{41}$ ", viendrait masquer que si le pardon délie et la promesse lie, ce ne sont pourtant pas les mêmes termes qui sont alors liés et déliés. Car si Hannah Arendt croit que la promesse sert à maîtriser l'imprédictibilité du cours de l'action en liant celle-ci à ses conséquences futures et que le pardon répond à l'irréversibilité des conséquences de l'action en la libérant de celles-ci, c'est qu'elle limite le pouvoir de déliaison du pardon au lien entre l'action et ses conséquences ${ }^{42}$.

Pour Ricœur, le pardon n'est toutefois possible que s'il délie l'agent de son acte, et non pas simplement un événement passé de ses conséquences futures ${ }^{43}$. Ricœur fonde cette possibilité sur une compréhension ontologique de l'homme qui voit son être "comme acte et comme puissance ${ }^{44}$ ». Contre la tentation de séparer l'agent de son acte en plaidant pour le "devenir autre» de l'agent à travers le temps, Ricœur affirme en effet que la possibilité du pardon repose sur la distinction entre l'effectuation de l'action et la capacité qui la sous-tend:

[La réponse] est à chercher, selon moi, du côté d'un découplage plus radical que celui supposé par l'argument entre un premier sujet, celui du tort commis, et un second sujet, celui qui est puni, un découplage au cœur de la puissance d'agir - de l'agency —, à savoir entre l'effectuation et la capacité que celle-ci actualise. Cette dissociation intime signifie que la capacité d'engagement du sujet moral n'est pas épuisée par ses inscriptions diverses dans le cours du monde. Cette dissociation exprime un acte de foi, un crédit adressé aux ressources de régénération $\mathrm{du} \mathrm{soi}^{45}$.

40. Ibid., p. 588-589.

41. Ibid., p. 635 .

42. Ibid., p. 632.

43. Ibid., p. 636-637.

44. Ibid., p. 639.

45. Ibid., p. 638. 
L'homme serait irréductible aux actions qu'il a effectuées, aux torts qu'il a commis, à l'ayant été, parce qu'il serait originairement disposition et capacité, homme capable tourné vers ce qu'il a à faire. Ricœur rappelle en ce sens que chez Kant, "[r]adical est le "penchant" au mal, originaire est la "disposition" au bien ${ }^{46}$ ", ce par quoi il faut entendre qu'en deçà de l'effectuation contingente d'une faute se trouve toujours déjà la disposition originaire d'être capable d'autre chose ${ }^{47}$. Ricœur en conclut que:

[s]ous le signe du pardon, le coupable serait tenu pour capable d'autre chose que de ses délits et de ses fautes. Il serait rendu à sa capacité d'agir, et l'action rendue à celle de continuer. C'est cette capacité qui serait saluée dans les menus actes de considération où nous avons reconnu l'incognito du pardon joué sur la scène publique. C'est enfin de cette capacité restaurée que s'emparerait la promesse qui projette l'action vers l'avenir. La formule de cette parole libératrice, abandonnée à la nudité de son énonciation, serait: tu vaux mieux que tes actes $^{48}$.

La véritable condition de possibilité du pardon est donc l'antériorité du caractère originaire de la capacité par rapport à la contingence des actes, antériorité qui ne doit rien à l'avant et à l'après du temps, mais qui est plutôt ontologique.

En quoi le pardon peut-il alors servir à une refiguration de l'expérience temporelle, s'il travaille à délier l'agent de son acte ? Est-ce à dire que le pardon n'aurait aucun effet sur un temps humain? Mais en redonnant à l'agent sa capacité, le pardon ne contribue-t-il pas à notre ouverture originaire vers l'avenir malgré l'ayant été des actions passées? Quel serait alors ce nouveau temps opéré par le pardon?

L'exemple de l'amnistie nous avait déjà mis sur la piste d'une compréhension temporelle du pardon. Si l'amnistie efface les traces et impose l'oubli du passé, c'est qu'elle empêche également l'établissement d'une certaine temporalité:

Mais le défaut de cette unité imaginaire n'est-il pas d'effacer de la mémoire officielle les exemples de crimes susceptibles de protéger l'avenir des erreurs du passé et, en privant l'opinion publique des bienfaits du dissensus, de condamner les mémoires concurrentes à une vie souterraine malsaine? En côtoyant ainsi l'amnésie, l'amnistie place le rapport au passé hors du champ où la problématique du pardon trouverait avec le dissensus sa juste place ${ }^{49}$.

Quel est alors ce rapport au passé «guidé par l'esprit du pardon ${ }^{50}$ » ? Avec le pardon, l'oubli n'équivaut pas à de l'amnésie, mais à un travail de

46. Ibid., p. 640.

47. Ricœur remarque la même antécédence de l'être capable par rapport à la faute ellemême dans le mythe adamique. Cf., ibid., p. 640.

48. Ibid., p. 642.

49. Ibid., p. 588-589.

50. Ibid., p. 589. 
deuil, en ce que le passé de la faute peut rester, mais sur le mode apaisé de l'ayant été qui est présent en portant la marque du ne plus. Il faut donc pouvoir oublier la faute passée, sans pour autant la perdre à titre d'héritage:

Faut-il aller jusqu'à "oublier la dette", cette figure de la perte? Oui, sans doute, dans la mesure où la dette confine à la faute et enferme dans la répétition. Non, pour autant qu'elle signifie reconnaissance d'héritage. Un subtil travail de déliement et de liement est à poursuivre au cœur même de la dette: d'un côté déliement de la faute, de l'autre liement d'un débiteur à jamais insolvable. La dette sans la faute. La dette mise à nu ${ }^{51}$.

À ce passé apaisé s'ajoute également un futur pour l'homme fautif, puisque le travail de deuil «sépare définitivement le passé du présent et fait place au futur ${ }^{52} »$. Grâce au pardon, le fautif n'est plus prisonnier de sa faute, et il peut alors être «rendu à sa capacité d'agir, et l'action rendue à celle de continuer ${ }^{53}$ ». Le pardon refigure ainsi entièrement l'expérience du temps de l'homme fautif: il lui donne son passé sous un mode apaisé, mais encore présent à titre d'héritage pour le futur, et libère son futur en le renvoyant à sa capacité d'agir. Passé et futur sont alors synthétisés de façon particulière. Le passé, ne fermant plus le futur de l'agir, reste tout de même présent à titre de ne plus et d'exemple pour le futur.

Jusqu'où faut-il alors étendre les refigurations de l'expérience temporelle que nous devons à la mondanéité ? Comme la narration, ne sont-elles que le fruit d'un travail du langage, la promesse, l'amnistie et le pardon appartenant aux actes de langage? Et les différences entre l'amnistie et le pardon, seul le second n'effaçant pas les traces bénéfiques du passé, ne laissaient-elles pas entendre que certaines expériences temporelles, et donc certaines pratiques du temps, seraient plus souhaitables, voire plus justes, que d'autres? N'y aurait-il pas une temporalité heureuse, non pas au sens cognitif d'une fidélité au passé ${ }^{4}$ ? Des considérations éthiques se trouventelles au seuil de notre expérience du temps?

Nous croyons qu'en regard à la fois de l'anthropologie suggérée dans La mémoire, l'histoire, l'oubli et de la transformation de l'expérience du temps selon les synthèses opérées par diverses médiations, la conclusion s'impose que certaines expériences temporelles doivent être mises en œuvre plutôt que d'autres. Les synthèses temporelles qui doivent être réalisées sont celles de l'homme capable, c'est-à-dire de l'homme qui porte la charge de son passé, notamment en son exemplarité pour qu'il sache comment agir à l'avenir, mais sans que ce passé lui ferme l'ouverture vers le futur nécessaire à sa capacité d'agir.

51. Ibid., p. 653 .

52. Ibid., p. 649.

53. Ibid., p. 642.

54. "[L'idée de la mémoire heureuse] était dissimulée dans la définition de la visée cognitive de la mémoire par la fidélité » (ibid., p. 643). 
Comme nous l'avons vu, plusieurs médiations pourraient participer à une telle temporalité, comme le pardon, le deuil et l'identité narrative. Et en deçà de ces médiations portées par le langage, c'est déjà dans les simples objets du quotidien que s'appuie la possibilité d'une telle temporalité. Car que serait l'agir sans la constance des objets, constance qui travaille notre expérience temporelle en la stabilisant déjà dans la durée? Nous y avons déjà fait allusion lorsque, rappelant le rôle de l'objet dans À l'école de la phénoménologie, nous faisions remarquer qu'il y sert de "fil d'Ariane dans le divers de la conscience». Ricœur évoque par ailleurs dans le même sens comment les objets et les lieux ouvrent la possibilité du souvenir:

C'est sur la surface de la terre habitable que nous nous souvenons avoir voyagé et visité des sites mémorables. Ainsi les "choses» souvenues sont-elles intrinsèquement associées à des lieux. Et ce n'est pas par mégarde que nous disons de ce qui est advenu qu'il a eu lieu. C'est en effet à ce niveau primordial que se constitue le phénomène des «lieux de mémoire », avant qu'ils deviennent une référence pour la connaissance historique. Ces lieux de mémoire fonctionnent principalement à la façon des reminders, des indices de rappel, offrant tour à tour un appui à la mémoire défaillante, une lutte dans la lutte contre l'oubli, voire une suppléance muette de la mémoire morte ${ }^{55}$.

Mais les objets et les lieux ne sont pas que des «indices de rappel» pour se souvenir. Ils le sont également pour l'agir de l'homme capable, car nos attentes, fondées sur des associations passées et des habitudes, sont éveillées par les lieux et les objets du quotidien. S’il est vrai que "[1]a mémoire corporelle peut être "agie" comme toutes les autres modalités d'habitude, telle celle de conduire une voiture que je tiens bien en main ${ }^{56}$ ", encore faut-il que l'objet - le volant de la voiture - se présente à nouveau pour que mon agir corporel puisse se réitérer et que je puisse dire savoir m'y prendre en tant qu'être capable. Dans l'hypothèse d'un monde environnant radicalement autre - nous pourrions penser au cas de l'exil ou au monde faisant suite à une catastrophe -, les habitudes passées conquises au contact des objets et des lieux ne pourraient plus être agies dans le futur, faute du retour des objets et des lieux qui les amorçaient. Dans ces circonstances, retrouver une temporalité où nous pourrions à nouveau être homme capable, c'est-à-dire notamment homme sachant s'y prendre, exigerait de se familiariser avec ce monde environnant étranger ${ }^{57}$. C'est seulement ainsi que nous pourrions acquérir, à travers la constance des objets et des lieux, un nouveau

55. Ibid., p. 49. Sur les objets comme indices de rappel. Cf., ibid., p. 46.

56. Ibid., p. 48.

57. Sur la rupture du monde familier et de sa normalité et l'acquisition graduelle d'un monde étranger, déjà dans sa structure générale de monde, Cf. Edmund Husserl, Autour des Méditations cartésiennes (I929-I932). Sur l'intersubjectivité [Hua XV], Grenoble, Jérôme Millon, I998, p. I36-4 I [210-4]; Edmund Husserl, Sur l'intersubjectivité II [Hua XIII-XIV-XV], Paris, Presses Universitaires de France, 200I, p. 342-344 [428-430]. 
passé chargé d'habitudes et d'attentes dignes d'asseoir une maîtrise du futur. Les lieux et les objets du quotidien et de la familiarité, par leur constance et leurs retours fréquents, travailleraient en ce sens également une temporalité heureuse.

Nous aimerions également souligner que ce détour par la narration, l'amnistie, la prescription, le pardon et les objets quotidiens nous incite à croire que nous n'avons évidemment pas couvert toute l'étendue des médiations pouvant intervenir pour transformer notre expérience du temps. Nous aurions pu prendre pour exemple le discours apocalyptique et ses effets sur le temps, ou celui de la "crise» comme discours établissant une nouvelle temporalité ayant ses effets sur l'homme capable. Tenir un discours «en temps de crise », cela n'équivaut-il pas à poser le passé en rupture? Dire qu'il y a crise, n'est-ce pas dire qu'il y a eu un changement ou un événement mettant en situation d'exception notre temps présent, le coupant ce faisant de tout autre temps? Et qu'en est-il du discours sur la fin des temps ${ }^{58}$ ? L'étendue des médiations travaillant notre temps pourrait donc être plus vaste. Mais le détour par l'œuvre de Ricœur devait surtout nous convaincre de deux choses. Nous pouvons être sensibles à des temps travaillés et transformés par plusieurs médiations objectives. Et ces temps peuvent avoir des conséquences éthiques et normatives pour notre pratique, comme le temps adéquat à l'homme capable devait nous le montrer.

\section{Conclusion}

Nous avons suivi Ricœur dans l'élaboration graduelle des différentes fonctions que les médiations objectives pouvaient avoir à l'égard de notre expérience temporelle. Ces fonctions se sont révélées être déjà présentes dès les articles portant sur la phénoménologie husserlienne. Ricœur remarquait le double rôle que jouait chez Husserl le pôle de la mondanéité, soit celui de révéler les couches intentionnelles de la conscience lors du retour réflexif vers l'ego, et celui de stabiliser la conscience dans des unités à titre de «fil d'Ariane pour le divers de la conscience". Il est possible selon nous de trouver une réappropriation de ces deux rôles chez Ricœur, notamment dans le concept de refiguration narrative de l'expérience. Car la narration refigure notre expérience non seulement en réitérant et révélant une expérience prénarrative, mais également en la synthétisant selon des unités pratiques cohérentes dans lesquelles s'intriquent passé, présent et futur éloignés. En empruntant la voie de la refiguration narrative de l'expérience, nous avons été finalement amenés à soupçonner des abus de la narration par rapport à notre expérience du temps. Car les médiations narratives ne servent pas toujours à stabiliser l'expérience temporelle, tel un fil d'Ariane dans le divers de

58. À titre d'exemple, les analyses de Michael Fœssel sur l'apocalypse: Michaël Fœssel, Après la fin du monde. Critique de la raison apocalyptique, Paris, Éditions du Seuil, 20I2, $294 \mathrm{p}$. 
la conscience. Elles contribuent aussi à transformer négativement cette expérience lorsqu'elles intriquent le passé, le présent et le futur de façon problématique - par exemple lorsqu'en commémorant trop le passé elles ferment toute ouverture à la nouveauté du futur.

Aidés de ces diverses fonctions de la médiation narrative, nous avons identifié des médiations non narratives de notre expérience temporelle dans l'amnistie, la prescription, la promesse, le pardon et les objets du quotidien. Chacune réalise de façon particulière une synthèse du temps: parfois en effaçant l'origine, parfois en maintenant présent le passé, mais sans la marque du "ne plus ", parfois de façon plus heureuse en gardant le passé avec sa charge d'exemplarité pour le futur, sans pour autant nier l'ouverture de celui-ci. La question "quel temps est mis en ouvre par l'amnistie, la promesse, le pardon, l'exil, etc. ?» n'obtient donc pas une réponse univoque. Et notre expérience du temps, loin de n'avoir que la simple forme d'une succession d'instants présents, est plutôt soumise à l'action de certaines médiations lui donnant des formes particulières.

Le glissement du rôle de la médiation objective, qui devait d'abord servir de guide positif et qui finalement prenait aussi les traits d'un abus potentiel, a fait intervenir de plus en plus de considérations normatives dans notre propos par rapport au temps. Puisque notre expérience du temps est transformée, et non pas simplement réitérée, par des configurations que nous pouvons produire, c'est aussi la dimension éthique du temps que nous configurons qui doit être mise de l'avant et réfléchie. Certaines expériences temporelles seraient plus heureuses, plus éthiques, que d'autres. En effet, comme l'homme ne peut être capable selon Ricœur que si l'exemplarité de son passé peut encore s'ouvrir à un projet futur, toute expérience temporelle n'est pas alors adéquate à l'homme capable. Ainsi formulé, le rapport entre l'expérience temporelle mise en œuvre par des médiations objectives et l'homme capable est même proto-éthique. L'intrication heureuse du passé, du présent et du futur devient une condition pour que l'homme puisse être rendu à sa capacité d'agir, et donc à sa potentialité éthique. L'homme ne s'ouvre à sa possibilité d'agir ou non de façon éthique que si une certaine expérience temporelle est configurée. S'il y a trop de mémoire, l'homme est réduit à ses actes passés et ne peut plus agir de nouveau; s'il y a trop d'oubli, il ne peut s'appuyer sur l'exemplarité de ses actes passés pour agir de nouveau. Car si nous pouvons bien changer quelque chose au temps, ce sont également nos propres possibilités qui sont en retour modifiées par le temps que nous faisons. 